\title{
Male Breast
}

National Cancer Institute

\section{Source}

National Cancer Institute. Male Breast. NCI Thesaurus. Code C33048.

One of the two, usually rudimentary, mammary glands in the male. 